\title{
Medical Expulsive Therapy in Acute Colic are We Justified?
}

\author{
Sekar Hariharasudhan, Deepak Ragoori, Sriram Krishnamoorthy, Sunil Shroff and \\ M.G. Rajamanickam*
}

\begin{abstract}
Department of Urology and Renal Transplantation, Sri Ramachandra Medical College and Research Institute, Porur, Chennai, India
\end{abstract}

\begin{abstract}
Medical expulsive therapy (MET) is one of the most common conservative modality of treatment offered by general practitioners for ureteric colic and calculi. In many occasions, such treatment may have beneficial effect, but might be counter-productive, if offered inappropriately. We report a case of unilateral pelvi-ureteric junction obstruction with a large, redundant, extra renal pelvis that was misinterpreted in ultrasonography as hydroureteronephrosis. The dilatation was assumed to be due to ureteric calculus and hence treated with MET, that resulted in forniceal rupture and urinoma. The aim of this article is to highlight the possible catastrophe that can result as a result of inappropriate administration of MET, especially in those patients treated based on empirical diagnosis of ureteric colic.
\end{abstract}

Keywords: Hydroureteronephrosis, medical expulsive therapy, pelvi-ureteric junction obstruction, ureteric colic.

\section{INTRODUCTION}

Medical expulsive therapy (MET) is one of the most common conservative modality of treatment offered by general practitioners for ureteric colic and calculi. The success of the treatment depends on the presence of stone, its size, its location, degree of impaction and its configuration. Majority of the complications of MET are secondary to an inadequate evaluation of hydroureteronephrosis. Hence, the clinician has to ascertain the exact cause of hydroureteronephrosis before offering the patient MET. Inappropriate or injudicious treatment using MET can sometimes be counterproductive. We report a case of unilateral pelvi-ureteric junction obstruction with a large, redundant extra renal pelvis that was misinterpreted in ultrasonogram as hydroureteronephrosis. The hydroureteronephrosis was assumed to be due to ureteric calculus and hence treated with MET, that resulted in forniceal rupture and urinoma. This report highlights the possible potential complication that can arise as a result of inappropriate usage of MET, especially in those patients in whom a conclusive diagnosis is not made.

\section{CASE REPORT}

A 36 year old male presented with fever and acute right loin pain associated with chills and rigors of one day duration. The pain was mainly localized to the right loin, radiating to right groin and right iliac fossa. He also had a history of burning micturition. He did not have any associated symptoms and had no significant present or past history. His haematological and urinary analysis was normal.

*Address correspondence to this author at the Department of Urology and Renal Transplantation, Sri Ramachandra Medical College and Research Institute, Porur, Chennai India; Tel: 00919444163163;

E-mail: dr_rajamanickam@yahoo.com
Ultrasonography of the abdomen was reported as significant right hydroureteronephrosis. He was treated with MET by a general practitioner, for a possible ureteric calculus; however no conclusive radiological investigation was done to confirm the diagnosis. Following MET, his pain further aggravated and he developed chills, rigors and vomiting. The pain did not subside despite heavy dose of analgesics and antispasmodics, and subsequently he was referred to a tertiary centre for further management.

He presented with fever and pain, localized to the right loin. He gave history of for two days fever associated with chills and rigors. On examination he was looking toxic with fever and tachycardia. He had voluntary guarding of the right hypochondrium and acute tenderness at the right renal angle, with a tender mass over the right loin.

Laboratory blood investigations revealed a TC -12635 cells/cu $\mathrm{mm}$ and other parameters were within normal limits. Ultrasonography of the abdomen revealed gross right hydronephrosis with no evidence of calculus. The ureter did not appear dilated. The right kidney contour was irregular with a hypoechoic rim of collection around the kidney, suggestive of perinephric collection.

Contrast enhanced computerised tomography (CT) scan of abdomen revealed gross right hydronephrosis with a dilated extra renal pelvis, suggestive of PUJ obstruction with forniceal rupture and perinephric collection. (Fig. 1) However, there was no evidence of any calculus.

After stabilizing the patient with antibiotic and supportive measures, he underwent cystoscopy and right double ' $\mathrm{J}$ ' stenting, following which he improved symptomatically. Post operative USG abdomen revealed considerable reduction of hydronephrosis. He was discharged with oral antibiotics and was advised a follow up visit after 4 weeks. Dismembered pyeloplasty (Fig. 2) with double J stenting was done after a month and he was advised regular follow up. 

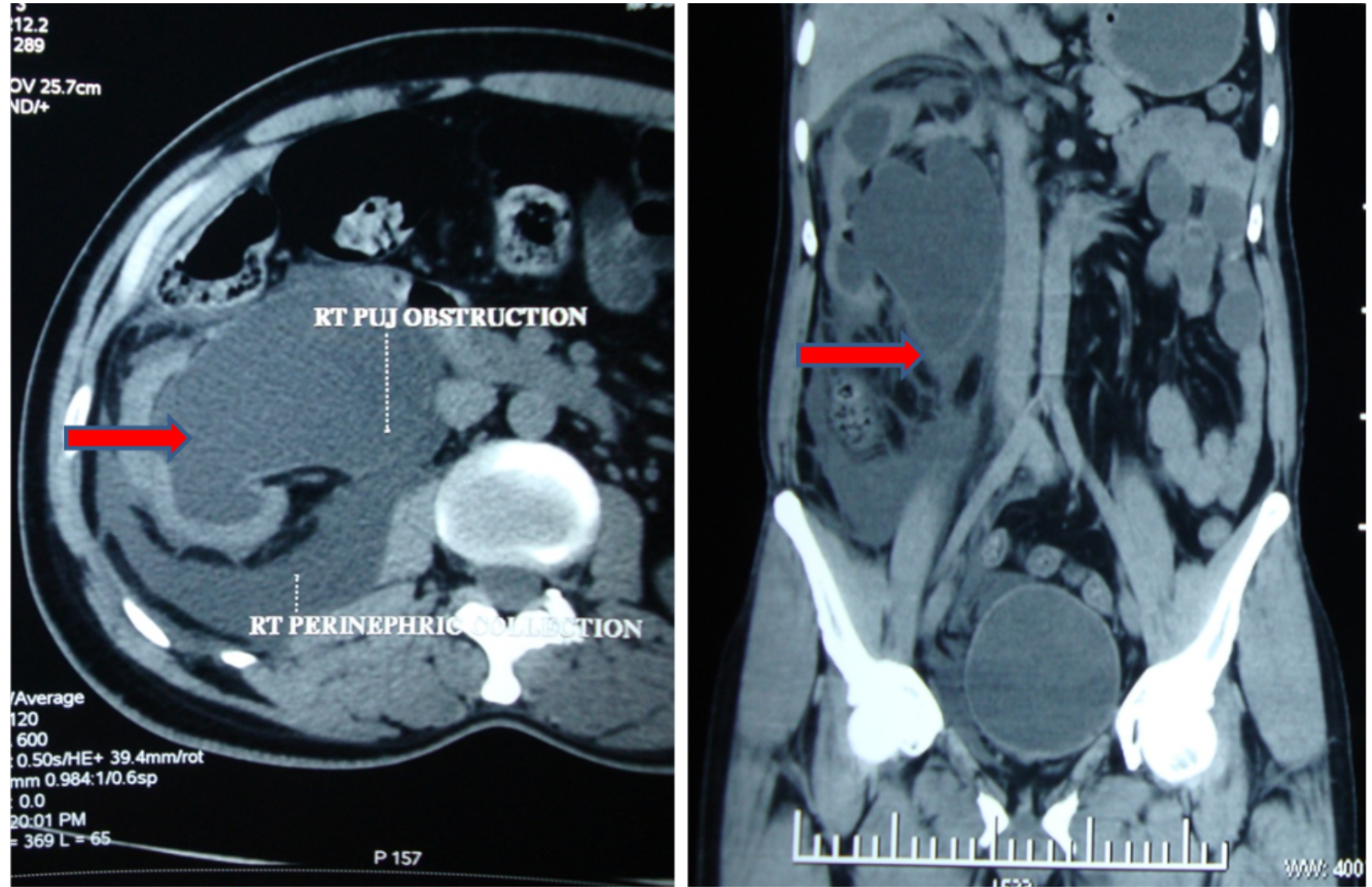

Fig. (1). Contrast enhanced CT abdomen - axial and coronal section showing the dilated pelvis and collecting system and the perinephric collection.

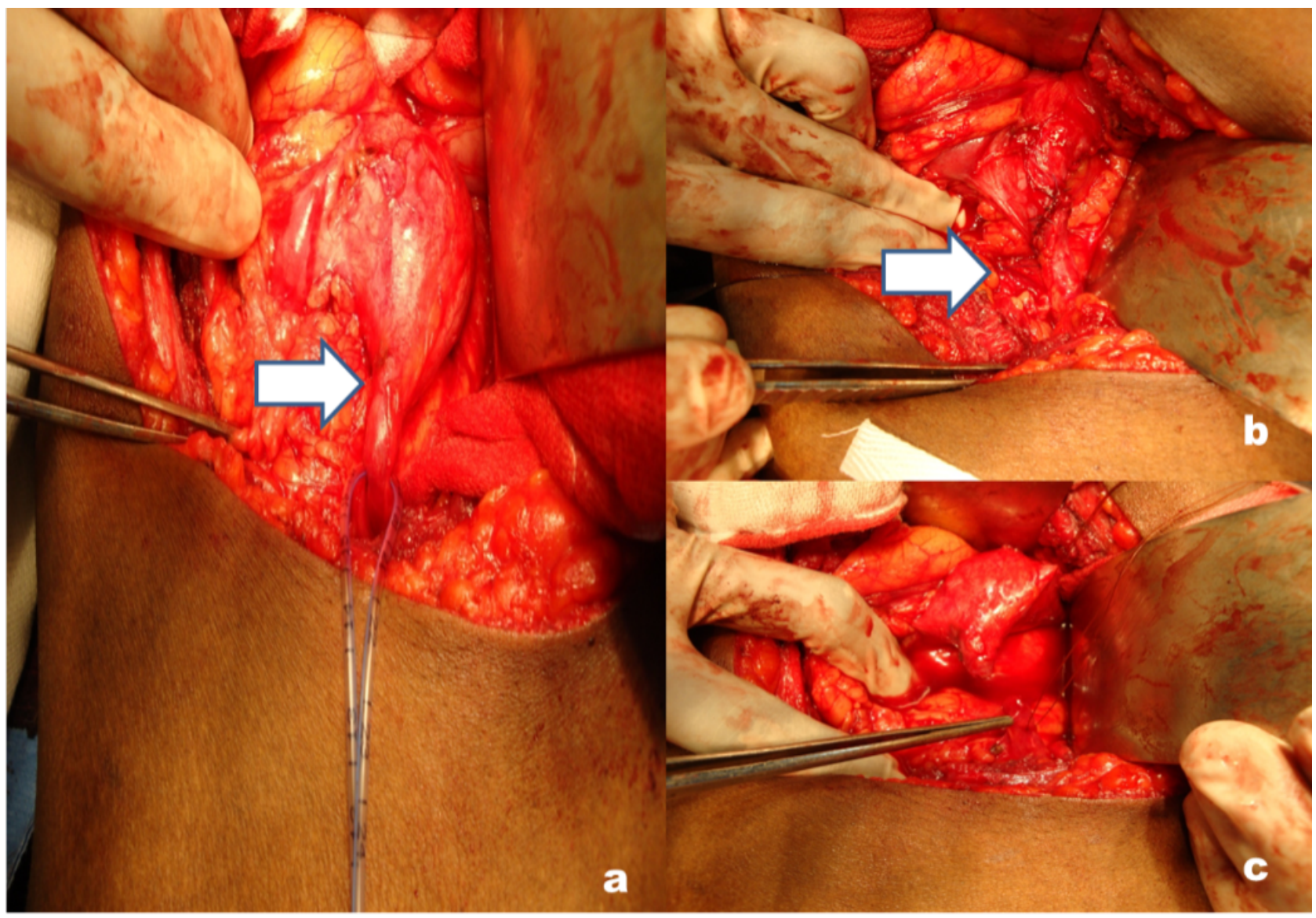

Fig. (2). (a) Pelvi-ureteric junction obstruction with a grossly dilated pelvis. (b, c) Open dismembered pyeloplasty in progress. 


\section{DISCUSSION}

There has been a paradigm shift in the concept of medical management for ureteric calculus and in patients presenting to the emergency department with ureteric colic.

The prevalence of stone disease is estimated to be around $2.3 \%$ [1]. With more than $20 \%$ of adults undergoing treatment for urinary stone disease, at some point of time in their lifetime [2], urolithiasis poses a significant health care problem in our country. As most of our patients initially present to the general practitioner before being referred to the tertiary care referral centre for definitive management, it becomes imperative for the practising general physicians to be aware of the appropriate management of patients with stone disease. Moreover, it should be remembered that not all patients who are presented with loin pain suffer from stone disease. Hence, it is even more important for the primary level physicians to be aware of the various potential conditions that mimic ureteric colic and treat them appropriately.

In our report we present one such condition, where a middle aged male was presented with colic. As the preliminary evaluation revealed hydroureteronephrosis, it was misinterpreted to be a stone disease and he was treated with I.V. fluids and diuretics, which resulted in forniceal rupture and urinoma.

Different modalities of conservative management have been adopted by different practitioners, which vary from increased oral fluid intake, administering intravenous fluids to intravenous diuretic therapy [3]. Such therapies that are aimed at increasing the urine output might theoretically facilitate spontaneous expulsion of the stone if not impacted; as there would be an increase in the intrarenal and intra ureteric hydrostatic pressure, that might mechanically propel the stone out. These treatments are aimed at a rapid stone elimination, as this would significantly reduce the duration of symptoms and promote early convalescence.

On the other hand, it might turn counterproductive, if the stone is impacted, as a sudden rise in the intraluminal hydrostatic pressure can cause rupture of urinary tract wall and or result in reversible or irreversible deterioration of renal function. Moreover MET cannot be given for a prolonged period. May et al. [4] had suggested that any MET should not be indiscriminately given beyond 4 weeks, as continuous treatment with MET would cause renal dysfunction, further pain and probably urosepsis.

There is very limited published data on the use of fluid therapy for patients with colic. Only one randomized controlled trial was identified during Pubmed search, which compares the use of diuretics or high volume fluid therapy for acute ureteric colic. Since not much data is available in literature, it becomes extremely difficult to identify how common the practice of diuretics or high volume fluid therapy is in our community.

At least in patients with stone disease, the renal parenchyma would be of reasonable thickness. Whereas, in patients with uretero-pelvic junction (UPJ) obstruction, and colic, it would be even more hazardous to offer diuretic therapy as the parenchyma would also be thinned out in such individuals, facilitating spontaneous forniceal rupture. As UPJ is also aperistalitic, any sudden increase in hydrostatic pressure can cause spontaneous urinoma [5]. Worster A. Richards [6] looked in to all the randomized controlled trials and quasi randomized control trials that had included diuretics or high volume fluid intake and inferred that there was no credible evidence supporting their role in colic management. Recent cochrane review concludes that there is no role for diuretic therapy and or high volume fluid therapy in acute ureteric colic (cochrane review 2009). However, a meta analysis by Hollingsworth et al. [7] found some benefit in those patients with stone disease, who were treated with MET.

This case report is mainly written to highlight the potential hazardous complications that can occur as a result of such indiscriminate use of medical expulsive therapy, as it would be impossible to know this with any degree of certainty unless we have published reports confirming these events.

\section{CONCLUSION}

Current evidence suggests that MET using calcium channel blockers or alpha antagonists with or without corticosteroids can be suggested as treatment for ureteral stones. More and more general practitioners prefer this modality of treatment as initial approach, in view of its expulsive efficacy, pain reduction and higher safety profile. This may be helpful in patients with stone disease, but might sometimes turn counterproductive if the colic is due to causes other than urolithiasis. This article highlights the fact that not all patients presenting with colic do suffer from stone disease and emphasis should be made to confirm the diagnosis of ureteral stones as the cause of colic before initiating MET, as an indiscriminate use of such therapy might sometimes lead to hazardous complications.

\section{CONFLICT OF INTEREST}

The authors confirm that this article content has no conflict of interest.

\section{ACKNOWLEDGEMENTS}

Declared none.

\section{REFERENCES}

[1] Gupta NP, Kesarwani P. Current approaches in the medical management of urolithiasis: A review article. Indian J Urol 2002; 19: 20-8.

[2] Ljunghall S, Lithell H, Skarfors E. Prevalence of renal stones in 60year-old men. A 10-year follow-up study of a health survey. Br J Urol 1987; 60: 10-3.

[3] Borghi L, Meschi T, Amato F, Briganti A, Novarini A, Giannini A. Urinary volume, water and recurrences in idiopathic calcium nephrolithiasis: a 5-year randomized prospective study. J Urol 1996; 155: 839-43.

[4] Liu M, Henderson SO. Myth: nephrolithiasis and medical expulsive therapy. CJEM 2007; 9: 463-5. 
[5] Seitz C, Liatsikos E, Porpiglia F, Tiselius HG, Zwergel U. Medical therapy to facilitate the passage of stones: what is the evidence? Eur Urol 2009; 56: 455-71.

[6] Worster A, Richards C. Fluids and diuretics for acute ureteric colic. Cochrane Database Syst Rev 2005: CD004926.
[7] Hollingsworth JM, Rogers MA, Kaufman SR, et al. Medical therapy to facilitate urinary stone passage: A meta-analysis. Lancet 2006; 368: 1171-9.

(C) Hariharasudhan et al.; Licensee Bentham Open.

This is an open access article licensed under the terms of the Creative Commons Attribution Non-Commercial License (http://creativecommons.org/licenses/by-nc/3.0/) which permits unrestricted, non-commercial use, distribution and reproduction in any medium, provided the work is properly cited. 